\title{
Otimização da pecuária nacional de forma sustentável
}

\author{
Optimization of the national livestock sustainably
}

\author{
NATEL, Andressa Santanna ${ }^{1 *}$; FAUSTO, Daiane Aparecida ${ }^{2}$; ARAGÃO, Thiago Ricielli \\ de Paula'; ABDALLA, Adibe Luiz ${ }^{1}$
}

\begin{abstract}
${ }^{1}$ Universidade de São Paulo, Centro de Energia Nuclear na Agricultura, Laboratório de Nutrição Animal, Piracicaba, São Paulo, Brasil.

${ }^{2}$ Universidade de São Paulo, Escola Superior de Agricultura Luiz de Queiroz, Piracicaba, São Paulo, Brasil.

*Endereço para correspondente: andressa.zoo@gmail.com
\end{abstract}

\section{RESUMO}

O objetivo deste trabalho foi avaliar o uso de nitrato de cálcio em substituição ao farelo de soja em dieta de ruminantes sobre a mitigação do metano entérico visando determinar os custos com a dieta do animal e os custos marginais de abatimento de carbono. $\mathrm{O}$ estudo foi realizado em duas fases, na primeira foi avaliado o potencial de mitigação de metano do nitrato por produção de gases in vitro. Os dados de mitigação de metano foram utilizados na segunda fase, que simulou uma propriedade leiteira com três sistemas de alimentação controle, sem nitrato, e sistemas com inclusão de 1,5 e $3 \%$ de nitrato na dieta. Foram utilizadas planilhas de cálculo para avaliar os custos e receitas de cada sistema com vendas de crédito de carbono resultantes da redução de metano ao final de 10 anos. O valor presente líquido (VPL) e a taxa interna de retorno (TIR) foram os índices utilizados na avaliação financeira. A venda de crédito de carbono não alterou o VPL, no entanto a inclusão de nitrato a 1,5 e $3 \%$ na dieta animal mostrou-se economicamente viável, pois reduziu o valor das dietas ( $\mathrm{R} \$ 5,89$ e 5,81/vaca/dia, respectivamente) em comparação com a dieta controle (R\$ 6,13/vaca/dia), além de contribuir para redução de gases de efeito estufa. Portanto, a suplementação de nitrato,como fonte de nitrogênio não protéico, em dietas para ruminantes, além de reduz a produção de metano possibilitou uma sensível redução no custo variável da atividade.

Palavras-chave: alimentação animal, emissão de gases, retorno econômico, ruminantes

\section{SUMMARY}

The objective of this work was to evaluate the calcium nitrate utilization in replacing to the soybean meal for ruminants diets under the mitigation of enteric methane aiming to determine the animal diet cost and the marginal costs for carbon rebates. The study was conducted in two stages, the first stage was in vitro gas production were performed in order to measure the enteric methane mitigation potential of the nitrate. The methane mitigation data were used in the second stage, which simulated a dairy farm with three feed systems: control system without nitrate, and systems with inclusion of 1.5 to $3 \%$ nitrate in the diet. The spreadsheets were used to evaluate the costs and revenues of each system resulting carbon credit sales of methane reduction in 10 years. The net present value (NPV) and the internal rate of return (IRR) were the indexes used in the financial evaluation. The carbon credit sale did not change the NPV significantly, however the inclusion of nitrate to 1.5 and $3 \%$ in animal feed showed economically feasible, because it reduced the diet value $(\mathrm{R} \$ 5.89$ e $\mathrm{R} \$ 5.81 / \mathrm{cow} /$ day, respectively) in comparison to control diet (R\$6.13/cow/day) besides contributing to the reduction and greenhouse gases. Therefore, the nitrate supplementation beside reduced the methane production, has the positive effect on the variable cost of the activity.

Keywords: animal feed, gas emissions, economic return, ruminants 


\section{INTRODUÇÃO}

O Brasil se destaca mundialmente por possuir o maior rebanho de bovinos comercial, são 212 milhões de cabeças (IBGE, 2013), A cadeia de ruminantes movimentou em $2015 \mathrm{R} \$ 169.3$ bilhões (CEPEA/USP, 2014), e gera aproximadamente sete milhões de empregos (IBGE, 2013). Contudo, a prática da pecuária causa impactos significativos ao meio ambiente, contribuindo para a depleção e poluição da água, perda da biodiversidade e intensificação das mudanças climáticas globais, em especial, a emissão de gases de efeito estufa - GEE (STEINFEL et al., 2006).

Em relação à emissão de dióxido de carbono equivalente $\left(\mathrm{CO}_{2}\right.$-eq), somente o setor de pecuária de leite emitiu em 2007 mais de 19 bilhões de toneladas (FAO, 2010), das quais cerca de 13 bilhões de toneladas foram atribuídas para o leite, contribuindo com $2,7 \%$ das emissões de GEE (IPCC, 2007). Estima-se que a emissão por unidade de leite produzido é de $2,4 \mathrm{~kg}$ de $\mathrm{CO}_{2}$-eq por $\mathrm{Kg}$ de leite corrigido para gordura e proteína na porteira da fazenda (FAO, 2010).

$\mathrm{O}$ dióxido de carbono $\left(\mathrm{CO}_{2}\right)$ e o metano $\left(\mathrm{CH}_{4}\right)$ contribuem com a maior quantidade das emissões de GEE. A emissão destes gases é medida em toneladas de $\mathrm{CO}_{2}$-eq e cada tonelada equivale a um crédito de carbono. Desta forma, considerando o tamanho do rebanho nacional, o setor pecuário tem alto potencial para desenvolvimento de negócios relacionados aos créditos de carbono, principalmente em relação à fonte de fermentação entérica (MOSS et al., 2000).
Entre os compostos químicos, a suplementação de nitrato em dietas para ruminantes tem sido estabelecida para a redução de metano (SILIVONG et al., 2011), pois inibe a metanogênese (VAN ZIJDERVELD et al., 2010), resultando em menor gasto energético ao animal (LENG \& PRESTON, 2010). O nitrato pode ainda ser utilizado como fonte de nitrogênio não proteico pelos animais (LI et al., 2013), o que poderia contribuir para o aumento da produtividade. Neste contexto objetivou-se avaliar a possibilidade de comercialização de crédito de carbono na pecuária, utilizando nitrato de cálcio em substituição ao farelo de soja(FS) como fonte de nitrogênio não proteico (NNP) em dieta de ruminantes para a mitigação do metano entérico, visando determinar os custos com a dieta do animal e os custos marginais de abatimento de carbono, onde os créditos são comercializados no mercado voluntário.

\section{MATERIAL E MÉTODOS}

A primeira fase do estudo foi desenvolvida no Centro de Energia Nuclear na Agricultura no período de Janeiro a Maio de 2014, a fase seguinte foi realizada entre Outubro a Dezembro de 2014.

$\mathrm{Na}$ primeira fase foi realizada uma avaliação do potencial de mitigação do metano pela técnica de produção de gases in vitro. Os valores de inclusão de nitrato de cálcio de 1,5 e 3\% na dieta, em base da matéria seca, foram considerados a partir de dados da literatura (HULSHOF et al., 2012; LI et al., 2013; El-ZAIAT et al., 2014) por apresentarem resultados satisfatórios para redução de metano e 
não apresentarem problemas a saúde do animal (metahemoglobolinemia), se adicionados gradualmente a dieta de ruminantes.

Para o cálculo de emissão de gases do rebanho foi utilizado um cálculo simplificado a partir do fator de emissão (IPCC, 2007).

Para a elaboração do estudo da segunda fase, adotou-se a metodologia descritiva, para um projeto de mercado de carbono voluntário, através da venda de certificados de emissão reduzida (CREs) aplicado à atividade de bovinocultura de leite, o valor presente líquido (VPL) e a taxa de retorno (TIR) foram os índices utilizados na avaliação financeira.
Os dados de custo e receita descritos foram simulados a partir do levantamento de dados em artigos, revistas e teses sobre bovinocultura leiteira.

$\mathrm{Na}$ primeira fase, foram três tratamentos distribuídos em delineamento experimental inteiramente casualizado sendo uma dieta base $(50 \%$ de feno de Coast Cross: $50 \%$ de concentrado) e três níveis de adição de nitrato $0 \% ; 1,5 \%$ e $3 \%$ (\% MS) em substituição ao farelo de soja em equivalente proteico a $0 ; 23,9$ e $48,1 \%$ mantendo as dietas isonitrogenadas $(15 \% \mathrm{~PB})$. O substrato incubado (dietas experimentais) foi moído em moinho tipo Willey (Marconi, Piracicaba, SP, Brasil) a $1 \mathrm{~mm}$ (Tabela 1).

Tabela 1. Ingredientes e composição química das dietas experimentais (\% MS)

\begin{tabular}{|c|c|c|c|}
\hline \multirow{2}{*}{ Items } & \multicolumn{3}{|c|}{ Dietas Experimetais $^{1}$} \\
\hline & $0 \%$ & $1,5 \%$ & $3 \%$ \\
\hline \multicolumn{4}{|l|}{ Ingredientes (\%) } \\
\hline Feno Coast Cross & 50,0 & 50,0 & 50,0 \\
\hline Milho grão & 35,2 & 37,2 & 39,3 \\
\hline Farelo Soja & 14,8 & 11,3 & 7,7 \\
\hline Nitrato & - & 1,5 & 3,0 \\
\hline \multicolumn{4}{|c|}{ Composição química $^{1}$ (em g / 100 g MS) } \\
\hline MS, $\%$ do alimento & 91,2 & 91,2 & 91,3 \\
\hline MO & 94,3 & 94,1 & 93,9 \\
\hline PB & 15,4 & 15,4 & 15,2 \\
\hline $\mathrm{EE}$ & 2,7 & 2,7 & 2,6 \\
\hline FDN & 50,3 & 50,2 & 50,2 \\
\hline FDA & 27,6 & 27,2 & 26,8 \\
\hline $\mathrm{NO}^{-2}$ & - & 1,00 & 2,01 \\
\hline
\end{tabular}

Foram utilizados quatro diferentes inóculos considerados como repetição e dois frascos por inóculo considerados como réplicas. Para cada inóculo, um branco (garrafa sem substrato, contendo apenas inóculo e meio de incubação) foi incluído em duplicata para correção dos dados de produção de gás e degradabilidade. Um padrão interno constituído de feno de Tifton 85 (substrato com produção de gás conhecida) foi incluído em duplicata por 
inóculo para monitorar as condições de incubação. Assim, para cada inóculo, foram incubadas 16 garrafas contendo os tratamentos experimentais, branco e padrão interno.

Os inóculos utilizados foram retirados de oito ovinos Santa Inês, machos, castrados, com peso médio de $62 \mathrm{~kg}( \pm 3,5 \mathrm{~kg})$, fistulados no rúmen e mantidos em confinamentos. Cada inóculo foi composto do líquido ruminal de dois doadores diferentes, totalizando quatro inóculos.Os animais eram alimentados individualmente com uma dieta composta por $50 \%$ de feno de Tifton (Cynodon spp) e $50 \%$ de concentrado com 18\% PB (milho moído, FS e mistura mineral), com adição de $1 \%$ de nitrato por grama de matéria seca ofertada com acesso livre a sal mineral e água. A alimentação foi fornecida duas vezes ao dia $(7: 00 \mathrm{~h}$ e 16:00h), em partes iguais. O período de adaptação foi de 14 dias até a coleta dos inóculos. A adição do nitrato na dieta dos doadores de inóculo deu-se para aumentar as bactérias redutoras de nitrato no líquido ruminal para essas competirem com as metanogênicas (JEYANATHAN et al., 2011).O inóculo de ovinos foi utilizado por ser o modelo animal com o qual o laboratório onde foi desenvolvida a primeira fase trabalhava.

Foi utilizada a técnica in vitro de produção de gases (THEODOROU et al., 1994) adaptada ao sistema semiautomático do Laboratório de Nutrição Animal Lana/CENA para avaliação da produção de metano in vitro (BUENO et al., 2005; LONGO et al., 2006), usando um transdutor de pressão e um data logger (Press Data 800, Lana, CENA / USP, Piracicaba, SP, Brasil).

Meio grama de cada dieta experimental foi adicionado em saquinho de determinação de FDN (\#F57 bags; 50 $\mathrm{mmx} 40 \mathrm{~mm} ; \quad 25 \pm 10 \mu \mathrm{m}$ pore size; ANKOM, Tecnology Corporation, Fairport, USA) e incubado em frascos de vidro de $160 \mathrm{~mL}$ de volume total e head space de $85 \mathrm{~mL}$, com $50 \mathrm{~mL}$ de meio de incubação (meio tampão THEODOROU descrito em PRESTON, 1994) e $25 \mathrm{~mL}$ de inóculo. Os frascos foram fechados, misturados manualmente, e incubados a $39^{\circ} \mathrm{C}$ em estufa de circulação forçada (Marconi MA35, Piracicaba, SP, Brasil) por $24 \mathrm{~h}$.

A pressão do gás foi medida às $2,4,8,16$ e $24 \mathrm{~h}$ de incubação. Para determinação da produção de metano, $2 \mathrm{~mL}$ de gás foram amostrados em cada tempo de mensuração de pressão, utilizando seringa de $5 \mathrm{~mL}$ e armazenados em tubo de vácuo de $10 \mathrm{~mL}$.

O volume total de gases foi calculado por uma equação de regressão pré-definida para as condições locais (Equação 1):

$\mathrm{V}=7,365 \times \mathrm{p}\left(\mathrm{n}=500 ; \mathrm{R}^{2}=0,99\right)$

Em que: $\mathrm{V}=$ volume de gás $(\mathrm{mL})$; e $\mathrm{p}=$ pressão mensurada (psi).

A produção de gás total (PGT), em $24 \mathrm{~h}$ de incubação, foi considerada como a somatória parcial da produção de gás em cada intervalo de tempo.

A concentração de metano foi determinada em cromatógrafo gasoso (Shimadzu GC-2014) acoplado a um detector de ionização de chama (FID) com coluna Shincarbon ST 100/120 micro empacotada $(1,5875 \mathrm{~mm} \times 1,0 \mathrm{~mm} \times$ $1 \mathrm{~m})$. As temperaturas de forno de coluna, injetor e detector de chama foram 60, 200 e $240{ }^{\circ} \mathrm{C}$, respectivamente. $\mathrm{O}$ gás de arraste foi o hélio a um fluxo de $10 \mathrm{~mL} / \mathrm{min}$. Concentrações de metano foram determinadas por calibração externa usando a curva analítica $(0,30,90$ e $120 \mathrm{~mL} / \mathrm{L})$ preparado com metano puro (White Martins PRAXAIR Gases Industriais Inc.; pureza 99,5mL/ L). A 
produção de metano $\left(\mathrm{PCH}_{4}\right)$ foi determinada de acordo com Longo et al. (2006), eq. (2):

$\mathrm{PCH}_{4}(\mathrm{~mL})=($ total gás + Head space $) *$ (concentração de $\mathrm{CH}_{4}$ )

Os valores de $\mathrm{PGT}$ e $\mathrm{PCH}_{4}$ foram expressos em função da matéria orgânica verdadeiramente degradada - MOVD $(\mathrm{mL} / \mathrm{g})$.

Os dados da primeira fase foram testados por modelo misto usando máxima verossimilhança restrita (Restricted Maximum Likelihood: REML)no procedimento MIXED do programa SAS ${ }^{\circledR}$ v93 (Statistical Analysis System Int., Cary, NC). O modelo inclui o efeito fixo da dose de nitrato e do inóculo e a interação entre dose de nitrato e inóculo. A variável resposta foi a média por frasco por inóculo. As médias foram obtidas pelo comando LSMEANS (SAS $\left.{ }^{\circledR}\right)$ Os efeitos significativos foram identificados pelo teste
F e teste de Tukey-Kramer. Foi declarada diferença estatística quando $\mathrm{P}<0,05$.

A segunda fase utilizou a metodologia descritiva e para realização dos cálculos foi utilizando o software de planilhas Microsoft Excel®. A análise das emissões de GEE consistiu na divisão do total de CO2-eq emitido pelo rebanho em um ano, pelo total de produto final produzido pelo rebanho. Portanto a unidade funcional adotada foi: $\mathrm{kg}$ de $\mathrm{CO} 2$-eq por $\mathrm{kg}$ de leite. As emissões de gases de efeito estufa para cada cenário avaliado foram calculadas utilizando os fatores default do IPCC (2007), e alguns fatores obtidos em estudos para as condições brasileiras (Tabela 2). O total de animais de cada categoria dentro do rebanho dos cenários prototípicos é multiplicado por estes fatores, obtendo-se a emissão total de cada gás do rebanho.

Tabela 2. Dados para o cálculo de emissão de $\mathrm{CO}_{2}$-eq

\begin{tabular}{|c|c|c|c|}
\hline Gás & Categoria & Fator de emissão & Unidade \\
\hline \multirow{4}{*}{$\mathrm{CH}_{4}$ entérico } & Touro & 64 & \multirow{4}{*}{$\mathrm{Kg} \mathrm{CH}_{4} /(\text { cab.ano })^{-1}$} \\
\hline & Vaca leiteira & 62 & \\
\hline & Vaca Seca & 42 & \\
\hline & Novilha (o) & 49 & \\
\hline \multirow{3}{*}{$\mathrm{CH}_{4}$ fezes } & Touro & 1 & \multirow{3}{*}{$\mathrm{Kg} \mathrm{CH}_{4} /$ (cab.ano $^{-1}$} \\
\hline & Vaca & 1 & \\
\hline & Novilha (o) & 0,5 & \\
\hline \multirow{2}{*}{$\mathrm{N}_{2} \mathrm{O}$} & Vaca leiteira & 0,030625 & \multirow{2}{*}{$\mathrm{t} \mathrm{N}_{2} \mathrm{O}(\text { cab.ano })^{-1}$} \\
\hline & Vaca seca & 0,0175 & \\
\hline $\mathrm{CH}_{4}$ & Resíduo- Leite pasteurizado & 3,3 & $\mathrm{Kg} \mathrm{CH} 4 /$ leite $\mathrm{t}^{-1}$ \\
\hline $\mathrm{CO}_{2}-\mathrm{eq}$ & Produção leite até porteira & 3,5 & $\mathrm{Kg} \mathrm{CO}_{2}$-eq/kg LCGP \\
\hline
\end{tabular}

Fonte: IPCC (2007); Alves \& Vieira (2010); EMBRAPA (2010a; 2010b).

A emissão total de GEE em cada sistema (eq. 3) foi calculada de acordo com os critérios do IPCC (2007), onde as emissões de $\mathrm{N}_{2} \mathrm{O}$ e $\mathrm{CH}_{4}$ emitidas em um ano foram convertidas em toneladas de
$\mathrm{CO}_{2}$-eq segundo dados obtidos no IPCC (2007) e somadas ao total de $\mathrm{CO}_{2}$ emitido pela produção do leite. Neste estudo não foram consideradas a produção de alimentos e outras atividades dentro da 
fazenda, necessárias para o manejo do rebanho.

$$
\mathrm{T}\left(\mathrm{CO}_{2}\right)=(\mathrm{X} 1 * 25)+(\mathrm{X} 2 * 298)+\mathrm{X} 3(3)
$$

Em que, $\mathrm{T}\left(\mathrm{CO}_{2}\right)=$ Emissão anual de $\mathrm{CO}_{2}$ emitido pelo rebanho de cada nível de adição de nitrato; $\mathrm{X} 1$ = Emissão anual de $\mathrm{CH}_{4}$ oriundo da fermentação entérica e fezes do rebanho em um ano; X2 = Emissão anual de $\mathrm{N}_{2} \mathrm{O}$ oriundo das fezes e urina do rebanho em um ano; e X3 = Emissão anual de $\mathrm{CO}_{2}$ das demais atividades (combustível, agricultura, adubação de pastagem - neste caso só foi considerada produção de leite até a porteira e a pasteurização do leite).

Posteriormente, obteve-se o balanço considerando a mudança do cenário pelo uso do produto nitrato em substituição a FS. O fator de redução do nitrato foi considerado apenas para a emissão de metano entérico. A redução de metano foi transformada em $\mathrm{CO}_{2}$-eq, conforme eq. (4):

$$
\text { Saldo de } \mathrm{CO}_{2} \text {-eq }=\mathrm{T}\left(\mathrm{CO}_{2}\right)-\Delta \mathrm{CH}_{4} * 21 \text { (4) }
$$

Em que, $\mathrm{T}\left(\mathrm{CO}_{2}\right)=$ Emissão total de $\mathrm{CO}_{2}$ do sistema; $\Delta \mathrm{CH}_{4}=$ Redução de $\mathrm{CH}_{4}$ pelo uso de nitrato de cálcio em substituição ao FS obtido pela avaliação in vitro; e 21 $=$ Fator de transformação da variável $\mathrm{CH}_{4}$ em $\mathrm{CO}_{2}$-eq (IPCC, 2007).

Então, dividiu-se o total de $\mathrm{CO}_{2}$-eq pela produção anual de leite corrigido para gordura e proteína (LCGP) para se obter a pegada de carbono de cada sistema, o que representa o $\mathrm{CO}_{2}$ liberado na atmosfera.

Para as análises econômicas foi simulada uma propriedade leiteira tecnificada, da bacia leiteira da região de Castro no Paraná. Foram consideradas 150 vacas holandesas em lactação com produção de leite $(\mathrm{kg} / \mathrm{vaca} / \mathrm{ano})$ de 22,25 , considerando a densidade do leite de 1,03 , e período de lactação de 230 dias com consumo de
$25 \mathrm{~kg}$ de Matéria Original/vaca/dia (83\% MS). O que resulta numa produção anual de $4783,5 \mathrm{~kg}$ de leite/ vaca/ lactação. A idade ao primeiro parto, porcentagem de vacas em lactação, descarte de vacas e mortalidade de bezerros foi de 36 meses, $75 \%, 6 \%$ e $0,5 \%$, respectivamente.

Para fins de cálculo de preço do leite e produção de $\mathrm{CO} 2$-eq/ L de leite corrigido para gordura e proteína (LCGP) utilizou-se os valores médio de gordura de 3,49, proteína 3,17 e CCS de 414.200 para a raça Holandesa, de acordo com estudo realizado por Ribeiro Junior et al.(2014) com propriedades na região de Castro, bacia leiteira do Paraná.

Para formação do rebanho, ainda foi considerado 50 novilhas e vacas secas com peso médio de $500 \mathrm{~kg}$ que foram mantidas em semi-confinamento, representando desta forma $15,6 \%$ do rebanho. As novilhas de 12 a 24 meses $(460 \mathrm{~kg})$ e as bezerras de até 12 meses $(340 \mathrm{~kg}$ ) foram mantidas em pasto mais suplementação e representaram 6,3 e $31,3 \%$ do rebanho, sendo composto, portanto por 20 e 100 animais, respectivamente.

A propriedade simulada conta com oito hectares, um sistema de ordenha a vácuo (Kepler Weber®, KW 900 L/M) com capacidade de seis vacas, sendo ordenhadas 48 vacas/ hora. Os animais em lactação são mantidos confinados em sistema de estábulo livre com acesso a área de lazer. A alimentação é fornecida em forma de dieta total no cocho conforme Tabela 3.

Os custos referentes às dietas foram calculados conforme o valor $(\mathrm{R} \$)$ da tonelada do ingrediente em matéria original (MO). Posteriormente, realizouse o cálculo do custo em $\mathrm{R} \$$ / vaca/ dia e o custo em $\mathrm{R} \$ / \mathrm{kg}$ de MO consumida/ vaca (Tabela 3). Para os cálculos do valor dos ingredientes da dieta foram utilizados 
dados de preço adaptados do CEPEA/USP (2014).

O preço do nitrato de cálcio é incerto, pois não é utilizado comumente como um aditivo em alimentação de ruminantes para mitigação de metano, é usado como adubo nitrogenado e seu preço pode variar largamente. $\mathrm{O}$ preço de referência do produto agrícola disponível (fórmula química: $\left.5 \mathrm{Ca}\left(\mathrm{NO}_{3}\right)_{2} \mathrm{NH}_{4} \mathrm{NO} 3,10 \mathrm{H} 2 \mathrm{O}\right)$ é de $\mathrm{R} \$ 4760,00$ a tonelada (Produto
Calcinit Yara, valor em 20 de Julho de 2016). Contudo, o produto a base de nitrato será utilizado em substituição à ureia e ao FS como fonte de NNP, trazendo $\mathrm{o}$ benefício da redução de metano entérico, assim optou-se em utilizar para base de cálculo o preço do nitrato de cálcio igual ao preço da ureia, uma vez que para o produtor a ureia será $o$ aditivo concorrente.

Tabela 3. Dieta das com níveis de inclusão de nitrato de cálcio para vacas leiteiras em confinamento

\begin{tabular}{|c|c|c|c|c|c|c|}
\hline \multirow{3}{*}{ Ingrediente $(\% \mathrm{MS})^{1}$} & \multicolumn{3}{|c|}{$\% \mathrm{MS}$} & \multicolumn{3}{|c|}{ kg de MO/vaca/dia } \\
\hline & \multicolumn{6}{|c|}{ Níveis de inclusão de nitrato de cálcio } \\
\hline & Controle & $1,5 \%$ & $3 \%$ & Controle & $1,5 \%$ & $3 \%$ \\
\hline Silagem de milho & 50,010 & 50,010 & 50,010 & 11,040 & 11,040 & 11,040 \\
\hline Milho moído & 10,730 & 17,015 & 29,170 & 2,370 & 3,510 & 4,500 \\
\hline Polpa cítrica & 10,730 & 10,730 & 10,730 & 2,370 & 2,370 & 2,370 \\
\hline Farelo de soja & 12,500 & 9,530 & 3,510 & 3,310 & 2,520 & 1,720 \\
\hline Farelo de algodão & 12,500 & 9,520 & 3,520 & 2,210 & 1,680 & 1,150 \\
\hline Ureia & 0,650 & 0,300 & 0,150 & 0,140 & 0,000 & 0,00 \\
\hline Nitrato & 0,000 & 0,015 & 0,030 & 0,000 & 0,330 & 0,660 \\
\hline Mineral & 2,180 & 2,180 & 2,180 & 0,480 & 0,480 & 0,480 \\
\hline Bicarbonato de sódio & 0,700 & 0,700 & 0,700 & 0,150 & 0,150 & 0,150 \\
\hline \multicolumn{4}{|c|}{ Custo Ração Total (R \$ kg Matéria Original) } & 0,280 & 0,270 & 0,260 \\
\hline \multicolumn{7}{|c|}{$\begin{array}{l}\text { Fonte: Resultados da pesquisa. } \\
{ }^{1} \text { Preço dos ingredientes }(\mathrm{R} \$ / \text { ton MO): Silagem de milho }(\mathrm{MS}): 120,00 ; \text { Milho grão }=395,00 ; \text { Polpa cítrica }= \\
395,00 ; \text { Farelo de soja }=930,00 ; \text { Farelo de algodão }=430,00 ; \text { Ureia }=1.489,00 ; \text { Nitrato }= \\
\text { 1.489,00; Bicarbonato }=1.200,00 ; \text { Suplemento mineral }=1.170,00 ; \text { Pastagem Coast Cross }(\mathrm{MS})=0,12 . \\
\text { Adaptado de CEPEA (2014). }\end{array}$} \\
\hline
\end{tabular}

A receita obtida através da venda do leite foi calculada utilizando o simulador para o cálculo de Valores de Referência do leite segundo a qualidade (CONSELEITEPARANÁ, 2014), com base no valor realizado no mês de setembro de 2014 de R\$ 0,9221 (Tabela 4). Já a receita pela venda dos terneiros e descarte foi calculada usando como base os preços sugeridos pelo
CEPEA/USP (Setembro, 2014) de R\$ 800,00 .

Para o planejamento econômico, custo e receita, foram utilizadas planilhas de cálculo do Microsoft Excel $\AA$. Os custos do sistema controle foram aplicados levando em consideração uma empresa/ fazenda do setor leiteiro e abordam os valores de implantação do sistema, custos fixos e custos variáveis para 
funcionamento do negócio. As receitas correspondem à soma dos valores apurados com a venda de leite e animais. Para base de cálculo nenhuma mudança foi aplicada nos cenários com adição de nitrato de cálcio, $1,5 \%$ e $3 \%$, os quais mantiveram as mesmas receitas, exceto no último ano onde foram vendidos os certificados de emissão reduzida (CREs). Quanto aos custos, apenas os custos variáveis foram alterados em função do valor da alimentação entre os sistemas, pela inclusão do nitrato de cálcio como fonte de NNP em substituição ao FS e retirada da ureia.

Tabela 4. Receitas advindas de um sistema padrão de produção de leite conforme

\begin{tabular}{lccc}
\hline Receita Anuais & R\$/und & Quant/ano & Total R\$/ano \\
\hline Leite (litro) $^{1}$ & 0,9221 & 1166400 & 1075537,44 \\
Terneiros + Descartes & & 79 & 63200,00 \\
\hline Total (R\$ /ano) & 800,000 & & 1138737,44 \\
\hline Fonte : Resultados da pesquisa & & \\
${ }^{1}$ Preço Leite: CONCELEITE-Pr (Setembro, 2014); ${ }^{2}$ CEPEA/USP, 2014.
\end{tabular}

Para análise de viabilidade do projeto em função da redução de GEE, procurou-se não entrar na elegibilidade do projeto pecuário quanto à geração de créditos de carbono, haja vista que os critérios para este fim não são bem definidos, assim como não foram feitos cálculos para a reforma de pastagem, outra forma de mitigação de gases. Portanto a análise foi realizada considerando apenas a redução de metano entérico pelo uso de nitrato na dieta dos animais. Contudo, projetos pecuários apresentam grande potencial de aprovação pelas entidades operacionais certificadas pelo Comitê Executivo da Conferência das Nações Unidas sobre Mudança do Clima, dada a sua capacidade de redução de emissão de metano aplicando métodos simples.

Para efeito de cálculo levou-se em conta o preço mínimo pago pela tonelada de $\mathrm{CO}_{2}-$ eq em leilões da BMF \& BOVESPA Edital no. 001/2012 (2012) de 2,70 $€ / \mathrm{tCO}_{2}$. Esse valor é do mercado voluntário, caso houvesse um projeto de
Mecanismo de Desenvolvimento Limpo (MDL), esses valores poderiam ser mais elevados. Dióxido de carbono equivalente é uma métrica utilizada para equalizar as emissões de vários GEE com base na relativa importância de cada gás, em relação ao $\mathrm{CO}_{2}$. Para a transformação em reais foi utilizado o valor do euro referente à cotação da primeira quinzena de outubro de 2014 de R \$ 3,198.

Para avaliação financeira do estudo foram utilizados os critérios de taxa interna de retorno (TIR) e valor presente líquido (VPL) (WESTON \& BRIGHAM, 2000). Em que o VPL é a diferença entre o valor presente das receitas menos o valor presente dos custos. Neste estudo, considerou-se a taxa mínima de atratividade (TMA) como $10 \%$ ao ano. Optou-se por utilizar a TMA de $10 \%$ ao ano, pois é a recomendada pelo Centro de Estudos Integrados sobre Meio Ambiente e Mudanças Climáticas do Ministério do Meio Ambiente (MMA, 2002), que trata da Proposta Revisada de Critério e 
Indicadores de Elegibilidade para a Avaliação de Projetos Candidatos ao MDL no Brasil.

A análise dos dados da segunda fase foi descritiva, usando a hipótese de um cenário padrão (sem inclusão de nitrato na dieta) comparando as mudanças deste cenário com outros dois, com inclusão de dose de 1,5 e $3 \%$ de nitrato na dieta. Todos os cálculos foram realizados utilizando o software de planilhas Microsoft Excel ${ }^{\circledR}$. Os resultados são apresentados como a média de valores econômicos e o potencial de redução de metano.

\section{RESULTADOS E DISCUSSÃO}

$\mathrm{Na}$ fase um, avaliação do potencial de mitigação de metano in vitro, após $24 \mathrm{~h}$ de incubação houve redução potencial de aproximadamente $50 \%$ na produção de metano quando comparada a dieta com $3 \%$ de nitrato (\%MS) com a dieta controle $(0 \%)$ (Tabela 5). Este resultado pode estar relacionado ao fato do nitrato agir como receptor de elétrons mais competitivo que o $\mathrm{CO}_{2}$, reduzindo a disponibilidade de $\mathrm{H}_{2}$ para as bactérias metanogênicas. Desta forma, quando o nitrato está presente no rúmen, o $\mathrm{H}_{2}$ é utilizado na redução de nitrato a nitrito a amônia, diminuindo assim a produção de metano (UNGERFELD \& KOHN, 2006). Outra hipótese é que a inibição da produção de $\mathrm{CH}_{4}$ pode ser atribuída à toxicidade direta de nitrito e outros produtos da redução de nitrato a amônia sobre as archeas metanogênicas (BOZIC et al., 2009; SAR et al., 2005), mas, no presente experimento, não foi mensurada a expressão genica das bactérias ruminais. Os resultados da PGT e MOVD não foram alterados $(\mathrm{P}>0,05)$ pela adição de nitrato em substituição a proteína da soja.

Tabela 5. Matéria orgânica verdadeiramente degradada (MOVD), matéria seca verdadeiramente degradada (MSVD) eprodução de gases total (PGT) e metano $\left(\mathrm{CH}_{4}\right)$ in vitro em dietas com nitrato de cálcio substituindo o farelo de soja

\begin{tabular}{|c|c|c|c|c|c|}
\hline \multirow{2}{*}{ Item } & \multicolumn{3}{|c|}{ Níveis de inclusão de nitrato de cálcio ${ }^{*}$} & \multirow{2}{*}{$\mathrm{EMP}^{*}$} & \multirow{2}{*}{$p$-valor } \\
\hline & Controle $\%$ & $1,5 \%$ & $3 \%$ & & \\
\hline MOVD, $\mathrm{g} / \mathrm{kg}$ & $551,7^{\mathrm{a}}$ & $534,1^{\mathrm{a}}$ & $549,5^{\mathrm{a}}$ & 13,22 & 0,77 \\
\hline MSVD, $\mathrm{g} / \mathrm{kg}$ & $566,5^{\mathrm{a}}$ & $561,4 \mathrm{a}$ & $576,8^{\mathrm{a}}$ & 12,53 & 0,59 \\
\hline PGT, mL/g MOVD & $106,3^{\mathrm{a}}$ & $103,7^{\mathrm{a}}$ & $101,2^{\mathrm{a}}$ & 1,97 & 0,84 \\
\hline $\mathrm{CH}_{4}, \mathrm{~mL} / \mathrm{g}$ DVMO & $17,4^{\mathrm{a}}$ & $12,3^{\mathrm{b}}$ & $9,3^{\mathrm{c}}$ & 1,47 & $<0,01$ \\
\hline $\mathrm{CH}_{4}, \%$ & $16,4^{\mathrm{a}}$ & $11,9^{\mathrm{b}}$ & $9,2^{\mathrm{c}}$ & 1,77 & $<0,01$ \\
\hline
\end{tabular}

*Médias seguidas de mesma letra na linha não diferem estatisticamente entre si pelo teste de Tukey a $5 \%$ de probabilidade; EMP = erro médio padrão.

Utilizando os fatores default do IPCC (2007) para gado de leite, foi calculada a quantidade de GEE produzidos no cenário padrão (sistema controle), os valores calculados foram: 27,$7 ; 0,46$ e 13,8 toneladas de $\mathrm{CH}_{4}, \mathrm{NO}_{3}-\mathrm{N}$ e $\mathrm{CO}_{2}$, respectivamente, corrigindo esses valores pela equação para $\mathrm{CO}_{2}$-eq (IPCC, 2007) 
foi observado um total de 843,3 toneladas de $\mathrm{CO}_{2}$-eq emitido pelo sistema controle (Tabela 6). A partir dos valores obtidos no sistema controle foram estimados os valores dos sistemas com inclusão de nitrato de cálcio pela equação de mudança de cenário (IPCC, 2007), sendo observada uma redução de 10,2 e 16,5 toneladas de $\mathrm{CO}_{2}$-eq/ano com a inclusão de 1,5 e $3 \%$ de nitrato de cálcio, respectivamente. Como o nitrato age exclusivamente no rúmen, esse percentual está relacionado apenas à redução de metano entérico pelos animais. Estes números representam uma significativa queda na emissão de $\mathrm{CO}_{2}$-eq, visto que no Brasil existem aproximadamente 930 mil produtores trabalhando no mercado de comercialização do leite (IBGE, 2013).

Tabela 6. Emissões de $\mathrm{CO}_{2}$-eq do sistema controlede alimentação ( $0 \%$ nitrato de cálcio) e sistemas com inclusão de nitrato de cálcio $(1,5 \%$ e $3 \%)$

\begin{tabular}{lcccc}
\hline \multirow{2}{*}{ Emissões/ano } & \multicolumn{3}{c}{ Níveis de inclusão de nitrato de cálcio } & \multirow{2}{*}{ EPM $^{2}$} \\
\cline { 2 - 4 } & Controle & $1,5 \%$ & $3 \%$ & \\
\hline Emissão, $\mathrm{tCO}_{2}$-eq/ano & 843,3 & 833,2 & 826,8 & 3,39 \\
Pegada de Carbono, $\mathrm{tCO}_{2}$-eq/t LCGP $(3,5$ e 4,5\%) & 0,56 & 0,55 & 0,54 & 0,004 \\
Redução $\mathrm{CO}_{2}$-eq (ton) & 0,00 & 10,2 & 16,5 & 3,38 \\
\hline
\end{tabular}

tCO2-eq: toneladas de dióxido de carbono equivalente; LCGP (3,5 e 4,5\%): leite corrigido para gordura $(3,5 \%)$ e proteína $(4,5 \%) ;{ }^{2} \mathrm{EPM}=$ erro padrão da média.

Para pegada de carbono foi observada queda suave entre os sistemas controle, $1,5 \%$ e $3 \%$ de nitrato correspondendo a $0,76,0,75$ e 0,74 t $\mathrm{CO}_{2}$-eq/t LCGP, respectivamente. Estes dados são relativos a uma produção anual de 1.310 toneladas de LCGP. O Brasil produziu em 2013 um total de 22,3 bilhões de litros de leite (IBGE, 2013) aproximadamente 20,8 bilhões L (LCGP, 3,5 e 4,5\%) resultando em uma emissão de cerca de 72,9 bilhões de tonelada de $\mathrm{CO}_{2}$-eq, desta forma os resultados do presente trabalho poderiam possibilitar uma redução na emissão GEE pela pecuária leiteira.

Uma vantagem ao produtor seria que embora essa prática de redução de metano entérica não seja preconizada pelo Plano ABC (Plano Setorial de Mitigação e Adaptação às Mudanças Climáticas Visando à Consolidação de uma Economia de Baixa Emissão de Carbono na Agricultura), se utilizada, pode vir a auxiliar o produtor na contratação de financiamento na área de reforma de pastagem ou implantação de tratamento de dejetos animais, aumentando assim o potencial de redução de $\mathrm{CO}_{2}$-eq de sua propriedade.

Com base na análise financeira, os custos totais diferiram entre os três sistemas de produção (Tabela 7), sendo maior no sistema controle $(0,74 /$ litro) e menor nos sistemas com 1,5 e $3 \%$ de inclusão de nitrato (0,72 e $0,71 /$ litro, respectivamente). Como os custos fixos se mantiveram iguais (R\$ 0,15/litro), pois nenhuma mudança foi aplicada nos custos fixos entre os sistemas avaliados, pode-se observar que a variação é devido ao custo variável, superior no sistema controle (R \$ 0,59/litro) em relação aos demais sistemas ( $\mathrm{R} \$ 0,57 /$ litro). Como o único diferencial entre os sistemas foi o custeio com a alimentação, substituição de 
FS por nitrato de cálcio e retirada da ureia, pode-se inferir que esta substituição foi economicamente viável. Desta forma, os menores custos nos sistemas onde o nitrato foi adicionado indicam que estes sistemas são mais sustentáveis, para análises de curto prazo.

Apesar de todos os sistemas apresentaram margem bruta positiva, os sistemas com inclusão de nitrato apresentaram melhores resultados, estes resultados indicam $\mathrm{o}$ impacto do custo de alimentação para o sistema, uma vez que o diferencial entre os sistemas era apenas a alimentação, em especial o custo com proteína advinda do FS. O custo com alimentação representa $50 \%$ dos custos operacionais de um sistema (LOPES \& CARVALHO, 2000), o que explica a variação da receita entre os sistemas, uma vez que a retirada da ureia e a substituição do FS pelo nitrato de cálcio, como fonte de NNP, reduziram os custos variáveis dos sistemas.

Tabela 7. Medidas de desempenho econômico da atividade leiteira em sistema controlede alimentação ( $0 \%$ nitrato de cálcio) e sistemas com inclusão de nitrato de cálcio $(1,5 \%$ e $3 \%)$

\begin{tabular}{|c|c|c|c|c|}
\hline \multirow{2}{*}{ Item } & \multicolumn{3}{|c|}{ Níveis de inclusão de nitrato de cálcio } & \multirow{2}{*}{$\mathrm{EPM}^{2}$} \\
\hline & Controle & $1,5 \%$ & $3 \%$ & \\
\hline Custo variável (R\$/ Litro leite) & 0,59 & 0,57 & 0,57 & 0,005 \\
\hline Custo fixo (R\$/ Litro leite) & 0,15 & 0,15 & 0,15 & 0 \\
\hline Custo total ( $\mathrm{R} \$$ / Litro leite) & 0,74 & 0,72 & 0,72 & 0,004 \\
\hline Receita bruta(R\$/ Litro leite) & 0,98 & 0,98 & 0,98 & 0 \\
\hline Margem bruta(R\$/ Litro leite) & 0,39 & 0,40 & 0,41 & 0,004 \\
\hline Margem líquida(R \$ Litro leite) & 0,24 & 0,26 & 0,26 & 0,005 \\
\hline Custo da alimentação/vaca/dia (R\$) & 6,13 & 5,89 & 5,81 & 0,067 \\
\hline Custo alimentação/Kgleite produzido (R\$) & 0,28 & 0,27 & 0,26 & 0,004 \\
\hline Receita do Leite, R\$/vaca/dia & 21,68 & 21,68 & 21,68 & 0 \\
\hline $\mathrm{RMCA}^{1}, \mathrm{R} \$ / \mathrm{vaca} / \mathrm{dia}$ & 15,60 & 15,85 & 15,93 & 0,071 \\
\hline RMCA, R\$/vaca/ano & $5.517,99$ & $5.610,10$ & $5.643,14$ & 0,072 \\
\hline
\end{tabular}

${ }^{\mathrm{T}} \mathrm{RMCA}=$ renda média por animal; ${ }^{2} \mathrm{EMP}=$ erro padrão da média.

A margem líquida não diferiu entre os sistemas com inclusão de nitrato de cálcio $(1,5$ e $3 \%)$, contudo foi maior que os valores observados no sistema controle (R\$ 0,26 /litro $\mathrm{x} \quad \mathrm{R} \$ \quad 0,24$ /litro, respectivamente), estes resultados indicam a estabilidade da atividade, podendo se manter em médio prazo.

Quanto ao custo com a alimentação podese observar que o sistema controle foi o que despendeu maiores custos com alimentação/vaca/dia, R \$ 6,13, seguido do sistema $1,5 \%$ e $3 \%$ de nitrato que despenderam $\mathrm{R} \$ 5,89$ e $\mathrm{R} \$ 5,81 / \mathrm{vaca} / \mathrm{dia}$ (Tabela 7). Quando esses custos são analisados em função da produção média diária de leite $(22,2 \mathrm{~kg} / \mathrm{vaca})$ os custos com alimentação representaram $0,28,0,27$ e $0,26 \mathrm{R} \$ / \mathrm{kg}$ leite/vaca para os sistemas controle, $1,5 \%$ e $3 \%$ de nitrato, respectivamente. Avaliando-se a redução dos gastos do sistema com inclusão de nitrato frente ao sistema controle tem-se uma redução de 3,6 e 7,1 \% nos gastos com alimentação/kg de leite, para os sistemas 1,5 e $3 \%$ de nitrato em 
comparação ao sistema controle, respectivamente, o que indica um aumento na RMCA com a inclusão de nitrato de cálcio na alimentação.

Em relação à quantidade de $\mathrm{CO}_{2}$ sequestrado e receitas com CREs, o sistema com 3\% de nitrato foi o que apresentou maior quantidade de $\mathrm{CO}_{2}$ sequestrado, 16,5 toneladas, $38 \%$ a mais que o sistema com $1,5 \%$ que reduziu 10,2 toneladas de $\mathrm{CO}_{2}$ em relação ao sistema controle. Usando-se de base a venda das CREs pelos valores obtidos no leilão da BMF \& BOVESPA (2012), 2,7 $€$ /tonelada de $\mathrm{CO}_{2}$-eq, os sistemas $1,5 \% \mathrm{e}$ $3 \%$ de nitrato obtiveram uma receita extra de 27,44 e 44,51 €/ano ou, R\$ 878,08 e R\$ $1.424,32$, respectivamente, num período de 10 anos, quando se estipulou que os créditos seriam vendidos (cotação do euro mês outubro de 2014, R\$ 3,20). Uma receita praticamente insignificante, porém, além do valor social, pode ser aplicada a venda de CREs por cooperativas no setor de pecuária leiteira ou como moeda de troca em empresas de insumos, estes valores podem tomar proporções expressivas.

Para avaliação do custo/ benefício nos projetos considerando os custos e a rotação dos sistemas em 10 anos, o VPL, mesmo sem a venda dos CREs, foi mais expressivo nos sistemas com inclusão do nitrato em substituição ao FS (R\$ $22.788,90$ e $\mathrm{R} \$ 68.555,72$ para sistema 1,5 e $3 \%$ de nitrato, respectivamente) em relação ao sistema controle (R\$ $111.175,17)$ e não diferiu com a venda das CREs. Porém, com o aumento da inclusão do nitrato na dieta (1,5 e 3\% MS) tanto o VPL (R\$ 22.788,90 e R\$ 68.555,72) como a TIR (14 e 17\%) se elevaram, permanecendo superior à taxa do custo de oportunidade do capital, o que significa que, considerando-se a TMA como $10 \%$ ao ano o aumento da inclusão de nitrato apresentou-se como alternativa financeiramente viável para o produtor, pois esse ganho está relacionado à maior RMCA (Tabela 8). Contudo, níveis elevados de nitrato na dieta animal podem trazer danos à saúde do animal (LENG et al., 2008).

Tabela 8. VPL e TIR dos sistemas controle e inclusão de 1,5 e $3 \%$ de nitrato de cálcio em 10 anos, sem e com a venda dos CERs no mercado voluntário a uma taxa de $10 \%$ a.a

\begin{tabular}{llccc}
\hline \multirow{2}{*}{ Crédito de comparação $^{1}$} & \multirow{2}{*}{ Aplicabilidade $^{2}$} & \multicolumn{3}{c}{ Sistemas } \\
\cline { 3 - 5 } & S/CREs & Controle & $1,5 \%$ & $3 \%$ \\
\hline VPL (R\$ano) & c/ CREs & $-111.175,17$ & $22.788,90$ & $68.555,72$ \\
\multirow{2}{*}{ TIR (\% a.a.) } & s/CREs & $-5 \%$ & $23.127,47$ & $69.104,82$ \\
& c/ CREs & $-5 \%$ & $14 \%$ & $17 \%$ \\
& & & $14 \%$ & $17 \%$ \\
\hline
\end{tabular}

${ }^{\mathrm{T}} \mathrm{VPL}=$ valor presente líquido; TIR: taxa interna de retorno $;{ }^{2} \mathrm{CREs}=$ certificado de emissões reduzidas.

Como a representação das CREs foi pouco significante para o montante do projeto, a viabilidade se deu pelo custeio com alimentação, em especial a redução da participação de farelo de soja na dieta total, uma vez que é um produto com alto valor de venda por competir com a alimentação humana. O sistema controle mostrou-se inviável, resultando em VPL e TIR negativos com valores de $\mathrm{R} \$$ - 
111.175,17/ano e $-5 \%$, respectivamente. Para mudar este cenário, uma alternativa seria a alteração da dieta ou o aumento da produtividade do rebanho o que acresceria a receita.

Analisando os resultados obtidos pelo VPL dos projetos com inclusão de nitrato de cálcio na dieta com as taxas de redução de $\mathrm{CO}_{2}$-eq, o produtor teria prioridade nas atribuições de incentivos/ financiamentos no programa ABC (MAPA, 2011) para recuperação das pastagens ou implantação de um sistema de compostagem, uma vez que ampliaria suas reduções.

É importante salientar que a emissão de metano por ruminantes, além de contribuir para a concentração de gases de efeito estufa (BEAUCHEMIN et al., 2008), representa uma perda de até $12 \%$ do consumo de energia bruta para o animal (JOHNSON \& JOHNSON, 1995; ECKARD et al., 2010). Segundo Kaharabata et al. (2000), uma vaca leiteira pesando, aproximadamente, $600 \mathrm{~kg}$ pode apresentar produção total variando de 268 a $450 \mathrm{~g}$ de $\mathrm{CH}_{4} /$ dia, sendo a energia perdida na forma de metano $(13,344 \mathrm{kcal} / \mathrm{g})$ suficiente para produzir 4,55 e $7,65 \mathrm{~kg}$ de leite com $4 \%$ de gordura, respectivamente. Assim, o incentivo a comercialização de crédito de carbono resulta em sistemas de produção mais sustentáveis e mais rentável ao produtor.

Conclui-se que a inclusão de nitrato de cálcio, como fonte de NNP em substituição ao farelo de soja, proporciona redução nas emissões de metano in vitro, e já bmtem sua eficácia de redução comprovada in vivo (LEE \& BEAUCHEMIN, 2014). Contudo, o elevado nível de sensibilidade dos resultados impede a afirmação de que o uso de nitrato na alimentação de ruminantes seja uma alternativa para a redução dos custos da dieta, uma vez que a incorporação de nitrato a dieta de ruminantes ainda não tem sua comercialização aprovada, os custos de comercialização do produto são incertos. Porém é importante salientar que a redução do $\mathrm{CO}_{2}$-eq nos sistemas representa um fator a mais para a aquisição de financiamento no plano de agricultura de baixa emissão de carbono.

\section{AGRADECIMENTOS}

Os autores agradecem ao Centro de Energia Nuclear na Agricultura, especificamente ao Laboratório de Nutrição Animal por disponibilizar suas instalações para a realização da primeira fase do estudo.

\section{REFERÊNCIAS}

ALVES, J.W.S.; VIEIRA, S.M.M. Segundo inventário brasileiro de emissão antrópicas de gases de efeito estufa relatório de referência: emissões de gases de efeito estufa no tratamento e disposição de resíduos. Brasilia: MCT, CETESB, 2010., p.100.

BEAUCHEMIN, K.A.; KREUZER, M.; O'MARA, F.; MCALLISTER, T.A.

Nutritional management for enteric methane abatement: a review. Australian Journal Experimental Agricultural, v.48, p.21-27, 2008.

BOLSA DE VALORES, MERCADORIAS E FUTUROS DE SÃO PAULO [BM\&FBOVESPA]. 2012. Leilão de Venda de Redução Certificada de Emissão (RCE). Disponível em: $<$ http://www.bmfbovespa.com.br/ConsultaLeiloes/leiloes-de-credito-de-carbonologin.aspx?idioma=pt-br $>$. Acesso em: 10 out. 2014. 
BOZIC, A.K.; ANDERSON, R.C.; CARSTENS, G.E.; RICKE, S.C.; CALLAWAY, T.R.; YOKOYAMA, M.T.; WANG, J.K.; NISBET, D.J. Effects of the methane-inhibitors nitrate, nitroethane, lauric acid, Lauricidin and the Hawaiian marine algae Chaetoceros on ruminal fermentation in vitro. Bioresource Technology, v.100, p.4017-4025, 2009.

BUENO, I.C.S.; CABRAL FILHO, S.L.S.; GOBBO, S.P.; LOUVANDINI, H.; VITTI, D.M.S.S.; ABDALLA, A.L. Influence of inoculum source in a gas production method. Animal Feed Science and Technology, v.23-124, p.95-105, 2005.

CENTRO DE ESTUDOS AVANÇADOS EM ECONOMIA APLICADA - CEPEA. 2014. Indicadores de preço. Disponível em: $<$ http://cepea.esalq.usp.br/indicador/>. Acesso em: 10 out. 2014.

\section{CONSELHO PARITÁRIO}

PRODUTORES/INDÚSTRIAS DE LEITE DO ESTADO DO PARANÁ CONSELEITE-PARANÁ. 2014. Valor de Referência para o Leite. Disponível em: $<$ http://www.conseleitepr.com.br/conseleite >. Acesso em: 20 out. 2014.

ECKARD, R.J.; GRAINGER, C.; KLEIN, C.A.M. Option for the abastement of methane and nitrous oxide from ruminant production: A review. Livestock Science, v.130, p.46-56, 2010.

EMPRESA BRASILEIRA DE PESQUISA AGROPECUÁRIA - EMBRAPA.

Relatórios de referência: emissões de metano por fermentação entérica e manejo de dejetos de animais. Brasília: MCT, 2010a. 120p.
EMPRESA BRASILEIRA DE PESQUISA AGROPECUÁRIA [EMBRAPA].

Relatórios de referência: emissões de óxido nitroso de solos agrícolas e de manejo de dejetos. Brasília: MCT, 2010 b. $106 \mathrm{p}$.

El-ZAIAT, H. M.; ARAUJO, R. C.;

SOLTAN, Y. A.; MORSY, A. S.;

LOUVANDINI, H.; PIRES, A. V.;

PATINO, H. O.; CORREA, P. S.;

ABDALLA, A. L. Encapsulated nitrate and cashew nut shell liquid on blood and rumen constituents, methane emission, and growth performance of lambs. Journal of Animal Science, v.92, p.2214-2224, 2014.

FOOD AND AGRICULTURE ORGANIZATION - FAO. Homepage da FAO. 2010. Disponível em: <www.fao.org > . Acesso em: 12 out. 2014.

HULSHOF, R.B.A.; BERNDT, A.; GERRITS, W.J.J.; DIJKSTRA, J.; VAN ZIJDERVELD, S.M.; NEWBOLD, J.N.; PERDOK, H.B. Dietary nitrate supplementation reduces methane emission in beef cattle fed sugarcane-based diets.

Journal of Animal Science, v.10, p.25274209, 2012.

INSTITUTO BRASILEIRO DE GEOGRAFIA E ESTATÍSTICA - IBGE. 2013. Indicadores estatística de produção pecuária, dezembro. 2013. Disponível em: $<$ http://.ibge.gov.br>. Acesso em: 16 out. 2013.

INTERGOVERNMENTAL PANEL ON CLIMATE CHANGE - IPCC. 2007.

Mudança do Clima 2007: a base das ciências físicas. Disponível em: $<$ http://www.mct.gov.br/upd_blob/0015/15 130.pdf $>$. Acesso em: 5 out. 2014. 
JEYANATHAN, J.; KIRS, M.; RONIMUS, R.S.; HOSKIN, S.O.; JANSSEN, P.H. Methanogen community structure in the rumens of farmed sheep, cattle and red deer fed different diets.

Microbiology Ecology, v.76, p.311-326, 2011.

JOHNSON, K.A.; JOHNSON, D.E. Methane emissions from cattle. Journal of Animal Science, v.73, p.2483-2492, 1995.

KAHARABATA, S.K.; SCHUEPP, P.; DESJARDINS, R.L. Estimating methane emissions from dairy cattle housed in a barn and feedlot using an atmospheric tracer. Environmental Science Technology, v.34, p.3296-3302, 2000.

LEE, C.; BEAUCHEMIN. K.A. A review of feeding supplementary nitrate to ruminant animals: Nitrate toxicity, methane emissions, and production performance. Canadian Journal of Animal Science, v.94, p.557-570, 2014.

LENG, R.A. The potential of feeding nitrate to reduce enteric methane production in ruminants. A Report to the Department of Climate Change.

Commonwealth Government of Australia, Canberra. Australia. 2008. 82 p. Disponível em: <http://www.penambulbooks. com/Downloads/LengFinal\%20Modified\%20\%2017-92008.pdf>. Acesso em: 15 nov. 2015.

LENG, R.A.; PRESTON, T.R. Further considerations of the potential of nitrate as a high affinity electron acceptor to lower enteric methane production in ruminants. Livestock Research for Rural Development, v.22, p.221, 2010.
LI, L.; SILVEIRA, C.I.; NOLAN, J.V.; GODWIN, I.R.; LENG, R.A.; HEGARTY, R.S. Effect of added dietary nitrate and elemental sulfur on wool growth and methane emission of Merino lambs. Animal Production Science, v.53, p.1195-1201, 2013.

LONGO, C.; BUENO, I.C.S.; NOZELLA, E.F.; GODDOY, P.B.; CABRAL FILHO, S.L.S.; ABDALLA. A.L. The influence of head-space and inoculum dilution on in vitro ruminal methane measurements. International Congress Series, v.1293, p.62-65, 2006.

LOPES, M.A.; CARVALHO, F.M. Custo de Produção do leite. Lavras: UFLA, 2000. 42p. (Boletim Agropecuário, 32).

MINISTÉRIO DA AGRICULTURA, PECUÁRIA E ABASTECIMENTO MAPA. Projeções do Agronegócio 2010/2011 e 2020/2021 e Plano de Agricultura de Baixa Emissão de Carbono (ABC). 2011. Disponível em: $<$ http://.agricultura.gov.br $>$. Acesso em: 15 out. 2014.

MINISTÉRIO DO MEIO AMBIENTE MMA. Critérios de elegibilidade e indicadores de sustentabilidade para avaliação de projetos que contribuam para a mitigação das mudanças climáticas e para a promoção do desenvolvimento sustentável. Brasília, 2002. 42p.

MOSS, A.R.; JOUANY, J.; NEWBOLD, J. Methane production by ruminants: its contribution to global warming. Annales de Zootechnie, v.49, p.231-253, 2000. 
RIBEIRO JUNIOR,J.C.; LIMA, J.B.A.; LEMOS, K.L. de; SILVA, L.C.C. da; TAMANINI. R.; BELOTI, V. Microbiota proteolítica e lipolítica de leite cru refrigerado da região nordeste e sul do Brasil. Semina: Ciências Agrárias, Londrina, v.36, n.6, suplemento 2, p.4289-4296, 2015.

SAR, C.; MWENYA, B.; SANTOSO, B.; TAKAURA, K.; MORIKAWA, R.; ISOGAI, N.; ASAKURA, Y.; TORIDE, Y.; TAKAHASHI, J. Effect of Escherichia coli wild type or its derivative with high nitrite reductase activity on in vitro ruminal methanogenesis and nitrate/nitrite reduction. Journal of Animal Science, v.83, p.644-652, 2005.

SILIVONG, P.; PRESTON, T.R.; LENG, R.A. Effect of sulphur and calcium nitrate on methane production by goats fed a basal diet of molasses supplemented with Mimosa (Mimosa pigra) foliage. Livestock Research for Rural Development v. 23, n.3, 2011.

STEINFEL, H.; GERBER, P.; WASSENAAR, T.; CASTEL. V.; ROSALES, M.; HAAN, C. Livestock's long shadow: environmental issues and options. Rome: FAO, 2006. 24 p.

THEODOROU, M.K.; WILLIAMS, B.A.; DHANOA, M.S.; MCALLAN, A.B.; FRANCE, J. A simple gas production method using a pressure transducer to determine the fermentation kinetics of ruminant feeds. Animal Feed Science and Technology, v.48, p.185-197, 1994.

UNGERFELD, E.M.; KOHN, R.A. The role of thermodynamics in the control of ruminal fermentation. In: SEJRSEN, K.; HVELPLUND, T.; NIELSEN, M.O.
(Editors). Ruminant Physiology:

digestion, metabolism and impact of nutrition on gene expression, immunology and stress. Wageningen, Netherlands: Wageningen Academic Publishers, 2006. p.55-85.

VAN ZIJDERVELD, S.M.; GERRITS, W.J.J.; APAJALAHTI, J.A.; NEWBOLD, J.R.; DIJKSTRA, J.; LENG, R.A.;

PERDOK, H.B. Nitrate and sulfate:

Effective alternative hydrogen sinks for mitigation of ruminal methane production in sheep. Journal Dairy Science, v.93, p.5856-5866, 2010.

WESTON, J.F.; BRIGHAM, E.F. Fundamentos da administração financeira. 10 ed. São Paulo: MAKRON Books, 2000. 1030p.

Data de recebimento: 05/01/2016

Data de aprovação: 29/07/2016 